\title{
OROFACIAL LESIONS AMONG DENTURE WEARERS
}

\author{
Khalid Al-Johani*
}

\begin{abstract}
Introduction: Removable dentures are being used as a replacement for missing teeth to restore function and aesthetics that has resulted in improved quality of life. However, different orofacial lesions may be presented as an associated complication to these appliances.
\end{abstract}

Aim: The present study aims at investigating the prevalence of denture-related lesions (DRLs) among the denture wearers.

Method: This was a descriptive, cross-sectional study. Clinical examination of 167 individuals with dentures was conducted from December 2013 to April 2014. Data form was designed to collect general information including personal data (e.g., age, gender), smoking practice, and frequency, duration and number of DRLs. The data were analyzed using the IBM SPSS software, version 22 (SPSS, Inc. IBM, Chicago, IL, USA). The responses were expressed as percentages.

Results: A total of 167 patients ( 82 men and 85 women) with a mean age of 58 years were enrolled in the present study. Of them, 84 had partial dentures while 75 had complete dentures. Eight patients had upper complete dentures and lower partial dentures. Different DRLs were reported. Sixty-six participants $(39.5 \%)$ had one or more denture-related abnormalities. Traumatic ulceration was the most common denture-associated lesions and was observed in 25 (15\%) participants.

Conclusion: DRLs were found to be more common among complete denture wearers than in partial denture wearers. However, regular follow-ups would help in early detection of the unnoticed abnormalities or complications of the dentures.

KEYWORDS: Dentures, denture-associated lesions, traumatic ulceration, orofacial lesions, denture wearers.

\section{INTRODUCTION}

An increase in the life span of the population has been observed due to improved medical and social conditions. However, in spite of the advancement in the dental services, there remain a significant number of patients, especially elderly patients, who seek teeth replacement; this has resulted in increased number of people who wear a removable prosthesis. Removable dentures, partial and complete, are used to replace the missing teeth to restore functions and aesthetics, resulting in improved quality of life (Preshaw et al., 2011). However, different orofacial lesions can present as complications toward the

* Assistant Professor, Department of Oral Diagnostic Sciences, Faculty of Dentistry- King Abdulaziz University 
working of these appliances. The complications include angular cheilitis, denture stomatitis, traumatic ulcers, and inflammatory hyperplasia. These lesions may arise as a result of physical, allergic or microbial factors associated with the denture. Physical injuries can result in traumatic ulcers and epulis fissuratum (Drake and Beck, 1993; Ellis et al., 2007; Farah et al., 2010; Preshaw et al., 2011).

Allergy can results in contact allergy to the denture base and microbial factors have been implicated in the denture stomatitis and angular cheilitis (Rashid et al., 2015).

Denture-associated erythematous candidiasisis a common inflammatory oral mucosal lesion that occurs on mucosa when it comes in contact with the fitting surface of a denture. The lesions are usually associated with candidiasis, other etiologic factors have been implicated including poor oral and denture hygiene, nocturnal denture wearing, and ill-fitting dentures (Lombardi and Budtz-Jorgensen, 1993; Farah et al., 2010).

Angular cheilitis is a chronic and erythematous fissuring of the angles of the mouth. The wrinkling in the commissure areas may cause saliva accumulation, and a chronic moist area may predispose to angular cheilitis. This is commonly present as a result of the loss of vertical dimension among the patients with dentures (Jafari et al., 2013).

Removable partial dentures are shown to increase the conditions of plaque, caries (especially root caries) and gingivitis, but no cases of periodontitis have been observed (Preshaw et al., 2011). Regular recall is essential to minimize the risk for dental caries and gingivitis.

Hence, the present study aims at examining and determining the prevalence of different dentureassociated orofacial conditions among the denture wearers.

\section{PATIENTS AND METHODS}

This is a descriptive cross-sectional study conducted on the denture wearers from December 2013 to April 2014. A special form containing three main sections was designed for recording all the relevant information. The first section included questions to explore the general information of patients(e.g., gender, age, education and medical history). The second part included information on the denture (type: complete or partial, cleaning practice).The third part included results of clinical examination including extra- and intraoral examination and detailed lesion descriptions (site, borders, color and configuration number, size and the types of lesions).

The diagnosis was based on the patient's history, clinical examination, and lesion character. The clinical examination of the oral lesions was performed by a calibrated clinician trained in recognizing oral lesions.

Oral hygiene status was assessed on the basis of cleaning methods and frequency.

The proposed study was reviewed by the Research Ethics Committee of the institution, and ethical approval was obtained for the same. None of the patients' identifications were revealed. The anonymous data were numerically coded and entered into the Statistical Package for Social Science (SPSS) version 22(SPSS Inc, Chicago, IL, USA) database and analyzed using the descriptive statistics and frequencies.

\section{RESULTS}

A total of 167 patients ( 82 men and 85 women) with a mean age of 58 years (range of 24-83 years) were enrolled in the present study (Table 1).

Of the present cohort, 107 patients had poor educational standard, and of these, 55 patients (33\%) were found to be illiterate, and $52(31.1 \%)$ had only an elementary level of education. In 
addition, 36 patients had attained high school, and 24 were university graduates.

TABLE (1) Age and gender of the present cohort.

\begin{tabular}{|c|c|c|c|c|}
\hline \multirow{2}{*}{ Age (Years) } & \multirow{2}{*}{ Male } & \multirow{2}{*}{ Female } & \multicolumn{2}{|c|}{ Total } \\
\cline { 3 - 5 } & & & No. & $\%$ \\
\hline $30-40$ & 5 & 3 & 8 & 4.7 \\
\hline $40-50$ & 18 & 11 & 29 & 17.4 \\
\hline $50-60$ & 8 & 17 & 25 & 14.9 \\
\hline $60-70$ & 10 & 21 & 31 & 18.6 \\
\hline $70-80$ & 5 & 4 & 9 & 5.4 \\
\hline 80 or more & 3 & 2 & 5 & 3.0 \\
\hline Total & 82 & 85 & 167 & \\
\hline
\end{tabular}

\section{Medical history:}

Table 2 shows the medical conditions in the present cohort of patients. The most commonly reported medical conditions were diabetes mellitus (16.8\%) and hypertension (16.2\%). Sixty-six patients $(39.5 \%)$ were smokers.

TABLE (2) Medical conditions among this group.

\begin{tabular}{|l|c|}
\hline Medical conditions & $\begin{array}{c}\text { No. of patients } \\
(\mathbf{n}=\mathbf{1 6 7})\end{array}$ \\
\hline Cardiovascular ailments & 5 \\
\hline Hypertension & 27 \\
\hline Anemia & 2 \\
\hline Diabetes mellitus & 28 \\
\hline Asthma & 2 \\
\hline Thyroid & 2 \\
\hline Hepatitis & 1 \\
\hline Others & 6 \\
\hline
\end{tabular}

\section{Orofacial findings:}

In the present cohort of patients, different denture-related mucosal lesions (DRLs) were reported. Sixty-six (39.5\%) patients were presented with one or more denture-related abnormalities.

Of this group of patients, 133 had both maxillary and mandibular dentures. In total, 21 and 13 patients had only maxillary and only mandibular dentures, respectively. Eighty-four patients had partial dentures while 75 had complete dentures. Eight patients had upper complete dentures and lower partial dentures. Oral lesions were common in females than in males, and in the older patients than in the younger patients.

Traumatic ulceration was the most common denture-related lesions and was observed in 25 $(15 \%)$ patients. It was found to be more common in the sixth decade of life (33.3\%) and was primarily associated with complete denture wearers than partial wearers (20\% and $11.9 \%$, respectively).

Denture-related stomatitis was found in $14.7 \%$ of complete denture wearers while $6 \%$ of removable denture had it with no significant difference (P value $>0.05$ ). Other reported lesions included angular cheilitis, flappy ridge, inflammatory hyperplasia and frictional keratosis (Table 3).

TABLE (3) Denture-associated lesions found in the present cohort of patients.

\begin{tabular}{|l|c|c|}
\hline \multicolumn{1}{|c|}{ Condition } & Frequency & Percent $(\%)$ \\
\hline Traumatic ulcers & 25 & 15 \\
\hline Denture-related stomatitis & 19 & 11.4 \\
\hline Angular chelitis & 7 & 4.2 \\
\hline Flappy ridge & 6 & 3.6 \\
\hline Inflammatory hyperplasia & 5 & 3 \\
\hline Frictional keratosis & 4 & 2.4 \\
\hline
\end{tabular}

Denture-related stomatitis most frequently affected the hard palate and was occasionally 
associated with angular cheilitis or median rhomboid glossitis (Figueiral et al., 2007).

Other mucosal conditions not related to the dentures, such as fissured tongue, atrophic tongue, geographic tongue, and smoker melanosis, were also reported in this cohort (Table 4).

TABLE (4) Frequency of the non-denture related mucosal lesions in the present cohort.

\begin{tabular}{|l|c|c|}
\hline \multicolumn{1}{|c|}{ Condition } & Frequency & Percent (\%) \\
\hline Fissured tongue & 33 & 19.8 \\
\hline Atrophic tongue & 6 & 3.6 \\
\hline Geographic tongue & 5 & 3.0 \\
\hline Hairy tongue & 3 & 1.8 \\
\hline Mucosal ulcer(s) & 2 & 1.2 \\
\hline Smoker melonosis & 1 & 0.6 \\
\hline Ankyloglossia & 1 & 0.6 \\
\hline
\end{tabular}

\section{DISCUSSION}

Oral health and the status of teeth have been found to affect the quality of life in many patients. Most individuals do not receive preventive dental services due to lack of awareness and also due to unavailability of preventive services in health centers. Many others opt for extraction and use partial or complete dentures to replace the missing teeth.

The occurrence of DRLs vary among the patients with prosthesis. In the present study,66 (39.5\%) patients were affected by DRLs, and this is similar to the results of other previously conducted studies (Jainkittivong et al., 2010).

Most of the patients of the present group had a low level of education, and this was in accordance with other studies that showed oral lesions to be more prevalent among patients with low level of education.
DRLs were observed to be higher in the case of complete denture wearers than in partial denture wearers, and this was in accordance with one of the previous study (Jainkittivong et al., 2010).

In addition, removable prosthesis has an effect on the oral mucosa as they may result in physical pressure or trauma that might lead to traumatic ulcers. Traumatic ulcers and dentures stomatitis were found to be the most common DRLs in the present study (25\% and 9.6 respectively), which was in accordance with Jainkittivong and coworker (2010).

Denture-related stomatitis is one of the most common DRLs. The reported prevalence of denturerelated stomatitis varies from $15 \%$ to over $70 \%$ depending on the population studied (Gendreau and Loewy, 2011). In the present study, denturerelated stomatitis affected about $9.6 \%$ of the study population and was mostly asymptomatic in nature or was often found to be associated with a burning sensation of the oral mucosa. This might be caused by the local irritation by dentures directly or as a result of the decrease in the salivary flow rate. However, other factors may predispose to denturerelated stomatitis, such as constant denture wear, the amount of tissue covered by dentures, low vitamin A levels, cigarette smoking, integrity of the prosthesis, and poor denture hygiene (Shulman et al., 2005; Freitas et al., 2008). Moreover, a previous study has reported the higher prevalence of DRS among the females (Figueiral et al., 2007).

Denture stomatitis has been classified into three subclasses (Newton's classification): (i) hyperemia (associated with trauma), (ii) generalized erythema, and (iii) (papillary hyperplasia; usually need surgery). They can also be categorized into (i) localized inflammation, with red spots around the salivary glands and diffuse palatal inflammation; (ii) diffuse hyperemic mucosa extending over the entire denture-bearing area, with a smooth surface; and (iii) hyperemic mucosa with a papillary or 
nodular surface usually localized to the central part of the hard palate (Budtz-Jorgensen and Bertram, 1970; National Center for Health Statistics, 1996; Shulman et al., 2005).

A recent meta-analysis study suggested that disinfection agents, antiseptic mouthwashes, natural substances with antimicrobial properties, microwave disinfection, and photodynamic therapy could be an adjunct or alternative to the antifungal medications in the treatment of denture-related stomatitis (Emami et al., 2014).

All DRLs (traumatic ulcer, denture hyperplasia, frictional keratosis, and candidiasis) were common occurrences in complete denture wearers. Inflammatory fibrous hyperplasia was significantly associated with complete denture use (Freitas et al., 2008); however, this was not common among the present cohort.

A variety of lesions not related to denture wearing were also found in the present cohort. The fissured tongue was the most common lesion not related to denture wearing (33 patients), and this was in accordance with a previously conducted study (Jainkittivong et al., 2010).

In addition, there was no association found between the systemic conditions(hypertension and diabetes mellitus) and DRLs, which may represent the prevalence of different systemic conditions in a society.

\section{CONCLUSIONS}

DRLs are common among denture wearers. The present study showed that DRLs were more common incomplete denture wearers than in partial denture wearers. Hence, regular follow-up is important for its early detection and management of any unnoticed abnormalities or complications in the prosthesis or any oral mucosal changes or lesions caused by the removable prosthesis. In addition, a dentist has to aim at improving the oral hygiene status and should stress on the oral hygiene measures. They need to educate the patients about the locally available cleaning agents that would further improve their oral hygiene.

Implant-supported prosthesis may also improve the retention and the oral health quality of life, and may result in decreased incidence of lesions.

The patients with oral lesions related to prosthesis may need treatment (for e.g., antifungal treatment, preprosthetic surgery, etc.) before the construction of any new prosthesis.

\section{DISCLAIMERS}

The author has no financial interests that may be relevant to the submitted work. This study was not presented as an abstract or a full paper in any national or international conferences.

\section{FUNDING SOURCE}

None.

\section{REFERENCES}

1. Budtz-Jorgensen E, Bertram U. Denture stomatitis. I. The etiology in relation to trauma and infection. ActaOdontol Scand. 1970; (28):71-92.

2. Drake CW, Beck JD. The oral status of elderly removable partial denture wearers .J Oral Rehabil. 1993;(20):53-60.

3. Ellis JS, Pelekis ND, Thomason JM. Conventional rehabilitation of edentulous patients: the impact on oral health-related quality of life and patient satisfaction. J Prosthodont. 2007;(16):37-42.

4. Emami E, Kabawat M, Rompre PH, Feine JS. Linking evidence to treatment for denture stomatitis: a meta-analysis of randomized controlled trials. J Dent. 2014;(42):99-106.

5. Farah CS, Lynch N, McCullough MJ. Oral fungal infections: an update for the general practitioner. Aust Dent J. 2010;(55Suppl 1):48-54.

6. Figueiral MH, Azul A, Pinto E, Fonseca PA, Branco FM, Scully C. Denture-related stomatitis: identification of aetiological and predisposing factors - a large cohort. J Oral Rehabil. 2007;(34):448-55. 
7. Freitas JB, Gomez RS, De Abreu MH, Ferreira E Ferreira E. Relationship between the use of full dentures and mucosal alterations among elderly Brazilians.J Oral Rehabil. 2008;(35):370-4.

8. Gendreau L, Loewy ZG. Epidemiology and etiology of denture stomatitis. J Prosthodont. 2011;(20):251-60.

9. Jafari A ; Lotfi-Kamran M ; Falah-Tafti A ; and Shirzadi S. Distribution Profile of Candida Species Involved in Angular Cheilitis Lesions Before and After Denture Replacement, Jundishapur Journal of Microbiology. 2013; (6): e10884.

10. Jainkittivong A, Aneksuk V, Langlais RP. Oral mucosal lesions in denture wearers. Gerodontology. 2010; (27):26-32.

11. Lombardi T, Budtz-Jorgensen E. Treatment of dentureinduced stomatitis: a review. Eur J ProsthodontRestor Dent 1993;(2):17-22.
12. National Center for Health Statistics. Third National Health and Nutrition Examination Survey, 1988-1994, Oral Examination Component. Hyattsville, MD, USA: United States Department of Health and Human Services (DHHS), Centers for Disease Control and Prevention, 1996.

13. Preshaw PM, Walls AW, Jakubovics NS, Moynihan PJ, Jepson NJ, Loewy Z. Association of removable partial denture use with oral and systemic health. J Dent. 2011;(39):711-9.

14. Rashid H, Sheikh Z, Vohra F. Allergic effects of the residual monomer used in denture base acrylic resins. European Journal of Dentistry. 2015 ;(9):614-619.

15. Shulman JD, Rivera-Hidalgo F, Beach MM. Risk factors associated with denture stomatitis in the United States. J Oral Pathol Med. 2005; (34):340-6. 